\title{
Lap Stapler Gastrojejunostomy with Total Truncal Vagotomy for Benign Gastric Outlet Obstruction.
}

\author{
Ashok Surybhanji Gajbhiye, Sanjay D. Dakhore, Ambrish P. Khathod
}

Address for communication:- Ashok Surybhanji Gajbhiye, Plot No.54, Kalpataru nagar, Besa Road, Manewada, Nagpur-34, email:-gsashok1972@gmail.com.Institute:- I.G.G.M.C, Nagpur-18, India.

\begin{abstract}
Aims: - To study the advantages and disadvantages of laparoscopic truncal vagotomy and gastrojejunostomy, the outcome of surgery in terms of - mean operative time, Conversion rate, Postoperative pain measurement, intraoperative and postoperative complication and duration of hospital stay. Methods: - A prospective analysis of operative, postoperative and short term outcome of 38 patients were carried out during time period of June 2008 to April 2013. Results: - Out of 38 patients, $73.68 \%$ patients were males and $26.31 \%$ patients were females. Male to female ratio was 2.8: 1. The mean operative time required was 108.21 minutes and the mean pain scale measured was $3 \pm 0.81$ on second day. The incidence of intraoperative and early postoperative complication was $5.26 \%$ and $10.52 \%$ respectively. The mean length of hospital stay was $6.78 \pm$ 2.59 days. Average follow up duration was $17.05 \pm 7.04$ months. Conclusion: - It is viable and safe option with shorter operative time and length of stay. It can be performed successfully with minimal morbidity and no mortality. However extreme care and skill is required to identify anatomy and handling of stapler.
\end{abstract}

Keywards: - Laparoscopy; Stapler Gastrojejunostomy; Vagotomy; Endoscopy.

\section{Introduction;-}

Inflammation, scarring or infiltration of the antrum and pylorus are associated with the development of Gastric Outlet Obstruction (GOO).Complications of Peptic ulcer disease (PUD) is bleeding [2, 5], perforation $[2,6]$ and gastric outlet obstruction [2, 7] remain prevalent. Gastric outlet obstruction is the more accurate term for the commonly used term 'Pyloric Stenosis', as the site of obstruction is rarely the pylorus itself. The obstruction is usually in the first part of the duodenum secondary to cicatrized duodenal ulcer or proximally where the diagnosis of carcinoma is most probable [8].

The role of the laparoscopic approach in the treatment of GOO is under investigation and may represent a valid form of therapy with low morbidity. The experience of several international centers has been published. One group in China performed laparoscopic truncal vagotomy and gastrojejunostomy for GOO related to PUD with nearly complete resolution of symptomatology. They reported no conversions to open procedure or mortalities [6].

The advent of Minimal Access Surgery allows the conventional procedure to be performed via laparoscopy. The minimally invasive technique continues to evolve. As technology and instrumentation continue to improve, so we have the complexity of operations that can be performed in a minimally invasive way.

\section{Material and Methods; -}

A prospective study of 38 cases of benign gastric outlet obstruction due to chronic duodenal ulcer, carried out during time period of June 2008 to April 2013. All these patients were selected from the general surgical units of our institute. All patients of benign gastric outlet obstruction due to chronic peptic ulcer disease were included in this study. Malignancy, chronic hypertrophic pyeloric stenosis, duodenal web, Bleeding disorder, peritonitis, portal hypertension, jaundice, Pregnancy and Patients unfit for general anaesthesia were excluded from this study. Endoscopy was done in all the cases to confirm the diagnosis [fig-1]. Upper endoscopy can help to visualize the gastric outlet and may provide a tissue diagnosis when the obstruction is intraluminal.

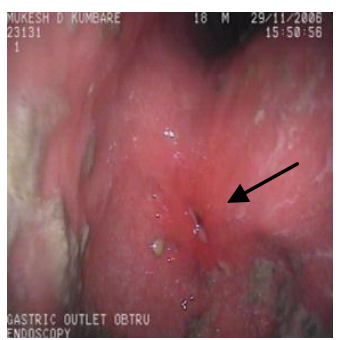

Fig 1-Endoscopic view of GOO (arrow). 
Operation was carried out by using standered technique as described by the author [7]. Under general anaesthesia, the patient was placed in modified lithotomy position. Port Position- The camera port $(10 \mathrm{~mm})$ is placed in the umbilicus. The right hand working port $(10 \mathrm{~mm})$ is placed in the left mid-clavicular line \& the left hand working port $(5 \mathrm{~mm})$ is placed in the right mid-clavicular line at the level of the umbilicus, depending on the level of the greater curvature of stomach. $5 \mathrm{~mm}$ port is placed in the epigastrium for retraction of the left lobe of liver. Another port in the left anterior axillary line $(5$ to $10 \mathrm{~mm})$ is placed for the Babcock's forceps that is used to retract the stomach. Anterior Vagotomy and biopsy-The left lobe of liver is retracted anteriorly while the stomach is pulled caudally. Posterior Truncal Vagotomy and biopsy-The left lobe is retracted anteriorly by a blunt nosed retractor introduced through the subxiphoid trocar. Proximally, the dissection was done bluntly to look for any additional branches, the so-called 'criminal nerve of Grassi'. Lower $5 \mathrm{cms}$ of the esophagus is cleared of all the attachments, by which the vagotomy becomes complete. Posterior Antecolic Gastrojejunostomy and Stapled gastro-jejunostomy: - The right mid clavicular port is converted to $12 \mathrm{~mm}$ for introducing Endo GIA stapler [fig-2].

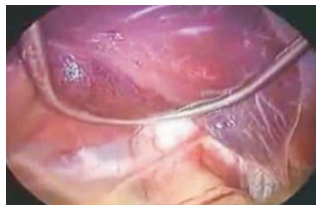

a)Exposure of esophagogastric junction.

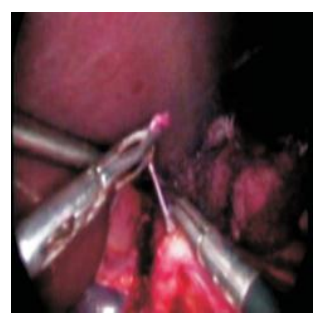

c) Division of vagus nerve for biopsy

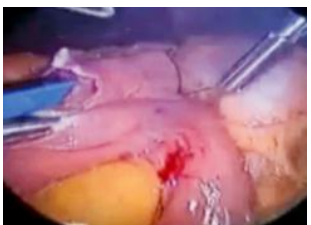

e) Firing of stapler.

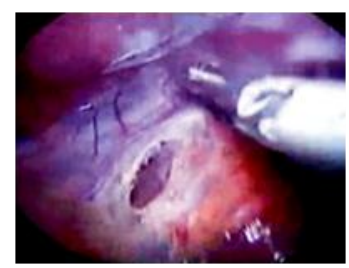

b) Opening of phrenoesophageal membrane.

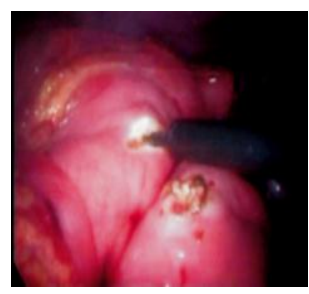

d) Stab incision over stomach and jejunum.

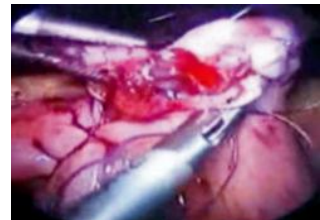

f) Closure of stapler entry wound using vicryl.

Fig-2) Technique of stapler GJTT (a-f).

\section{Results: -}

Present study was carried out under the aegis of a Medical College and Hospital from June 2008 to April 2013. 38 patients of gastric outlet obstruction due to chronic duodenal ulcer were included in this study. $15.78 \%$ patients were in age group 10-20yrs, 21-30yrs \& 31-40yrs each, $42.10 \%$ patients were in age group 41$50 \mathrm{yrs}, 10.52 \%$ were in age group $51-60.73 .68 \%$ patients were males and $26.31 \%$ patients were females. Male to female ratio was 2.8: 1 [fig.3].

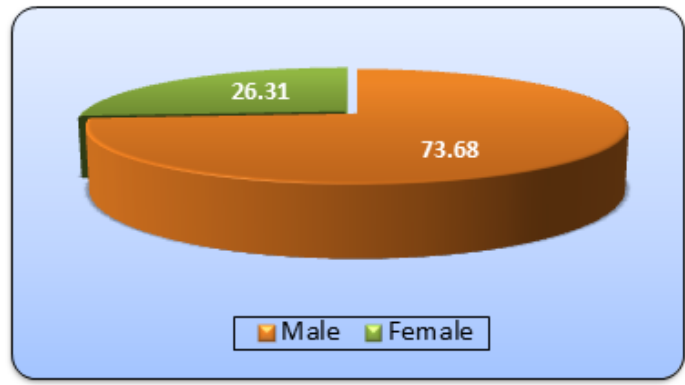

Fig-3] Pie diagram showing sex ratio. 
Among the selected patients, $94.73 \%$ patients were not operated previously for either peptic ulcer or its complication, $5.26 \%$ patient were operated previously for perforation peritonitis i.e. peptic ulcer related complication.

TABLE - 1] DISTRIBUTION OF SYMPTOMS

\begin{tabular}{|l|l|l|l|}
\hline Sr. No. & \multicolumn{1}{|c|}{ Symptoms } & No. of Patients & Percentage \\
\hline 1 & Pain in abdomen & 28 & $73.68 \%$ \\
\hline 2 & Vomiting(recurrent, nonbillious) & 38 & $100 \%$ \\
\hline 3 & Loss of appetite & 30 & $78.94 \%$ \\
\hline 4 & Loss of weight & 24 & $63.15 \%$ \\
\hline 5 & Post prandial fullness & 14 & $36.84 \%$ \\
\hline
\end{tabular}

Recurrent nonbillious vomiting was observed in all the patients. Among the selected patients, $73.68 \%$ were having pain in abdomen, $78.94 \%$ were having loss of appetite, $63.15 \%$ were having loss of weight and $36.84 \%$ were having postprandial fullness.

TABLE - 2] DISTRIBUTION OF SIGNS

\begin{tabular}{|l|l|l|l|}
\hline Sr. No. & Signs & No. of Patients & Percentage \\
\hline 1 & Pallor & 18 & $47.36 \%$ \\
\hline 2 & Abdominal distension & 6 & $15.78 \%$ \\
\hline 3 & Visible gastric peristalsis & 20 & $52.63 \%$ \\
\hline 4 & Succession splash & 38 & $100 \%$ \\
\hline
\end{tabular}

Succession splash was the commonest sign observed in all the patients. $47.36 \%$ patients were having pallor, $15.78 \%$ were having abdominal distension, $52.63 \%$ were having visible gastric peristalsis, and no patient was having palpable mass in the selected patient.

The mean operative time required was 108.21 mins. Among all selected patients $5.26 \%$ required time less than 90 min, $15.78 \%$ between $91-100 \mathrm{~min}, 26.31 \%$ between 101-110 min,21.05\% between 111-120 min, $15.78 \%$ between $121-130 \mathrm{~min}, 10.52 \%$ between $131-140 \mathrm{~min}$ and $5.26 \%$ patients required time more than 140 mins. Time required for surgery increased with difficulty in dissection. The conversion rate of laparoscopic gastrojejunostomy with total truncal vagotomy to open method was $2.63 \%$ i.e. out of 38 cases which were started as laparoscopic method; one patient case was converted into open method. The reason for conversion was due to adhesions [fig-4].

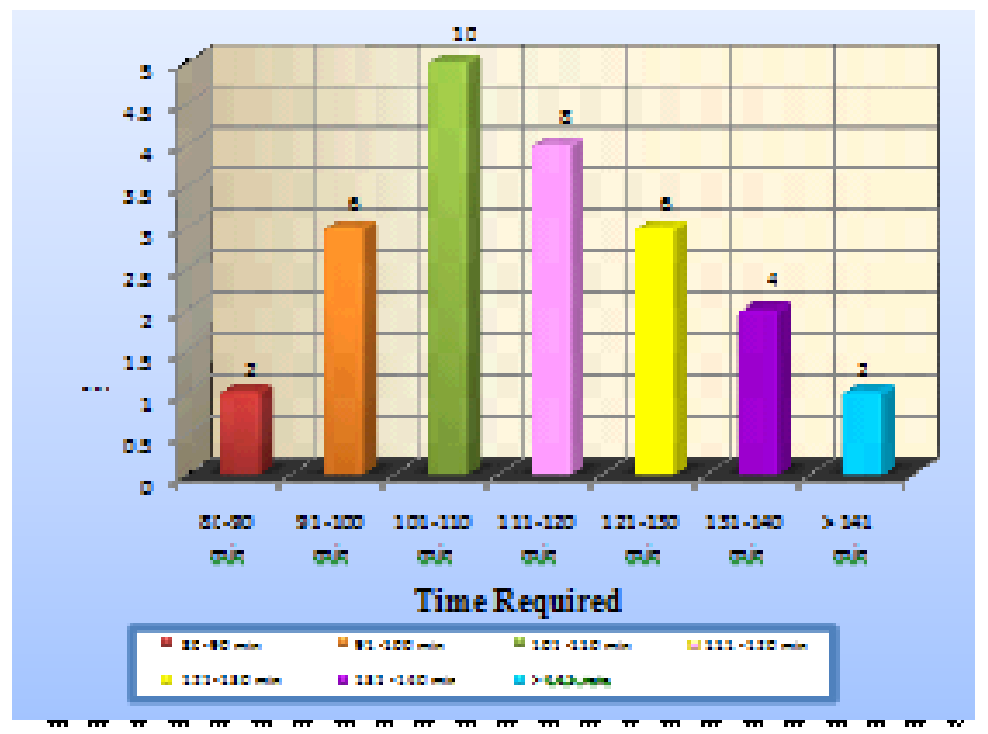

Fig-4] BAR DIAGRAM SHOWING OPERATVE TIME.

The post operative pain was graded on a scale of 0-10 as per the Visual Analogue Scale (VAS).The pain scale was calculated on day 1, 2 and 7 of operation. Thus on day 1, the mean pain scale measured was 5.26 \pm 1.19 . Thus on day 2 , the mean pain scale measured was $3 \pm 0.81$ and on day 7 , the mean pain scale measured was $0.36 \pm 0.59$. 


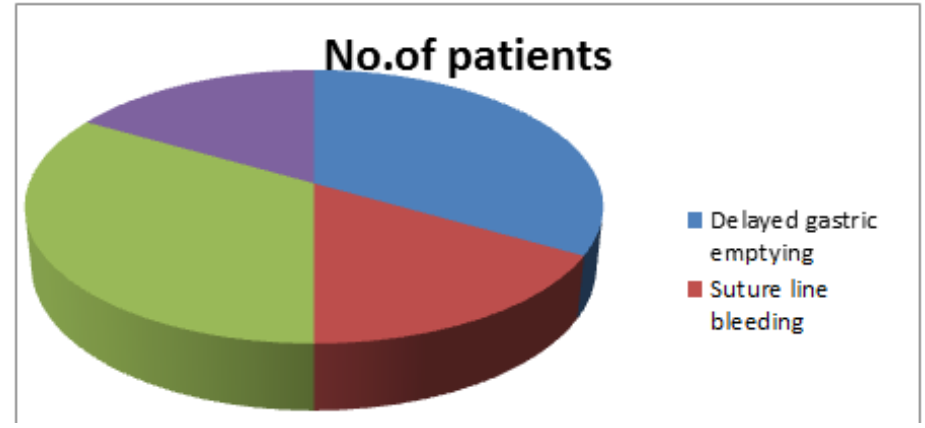

Fig-5] PIA DIAGRAM SHOWING POSTOPERATIVE COMPLICATIONS.

In the present study, two patients had intraoperative complication of suture line bleeding. Delayed gastric emptying was noted in four patients as an early postoperative complication. Thus the incidence of intraoperative and early postoperative complication was $5.26 \%$ and $10.52 \%$ respectively. In present study, six patients developed delayed postoperative complications. Four of them had postvagotomy diarrhoea and two had stromal ulceration. Thus the incidence of delayed postoperative complication was $15.78 \%$ [fig-5].

Patients required analgesics for mean of $46.73 \pm 41.94$ hours. The mean time for starting oral feeds was $4.73 \pm 2.42$ days. The mean time required for ambulation was $8.42 \pm 2.36$ hours. The mean length of hospital stay was $6.78 \pm 2.59$ days in the present study.

In the present study the rate of post operative wound infection was low. There was no major wound related complication in any of the patients. Only 1 patient had wound infection. Patient had only erythema and induration. Patients with wound infection were treated with antibiotics and local measures.

Subjective cosmetic results were assessed by patients themselves and showed that a large number of patients were satisfied with the quality of scars, they got after laparoscopic gastrojejunostomy and total truncal vagotomy. Only two patients was not satisfied with the quality of scars. In $78.94 \%$ patient scar was barely visible. A small well healed scar was present in $21.05 \%$ patients. No patient had either large well healed scar or complicated scar. Average follow up duration was $17.05 \pm 7.04$ months in the present study,

\section{Discussion: -}

Laparoscopic surgery is a well established alternative to open surgery across disciplines. Although the magnitude of impact varies by procedure, generally the benefits of laparoscopy on postoperative pain, cosmetics, hospital stay and convalescence are recognized widely.

The mean age of selected patients was 37.89 yrs. For females it was 36.4 yrs and for males it was 38.42 yrs. The mean age of patients in the study by $[3,4,6]$ was 40 yrs, 55.2 yrs and 42.2 yrs respectively. In the present study, 28 patients were males and 10 patients were females. Out of 63 patients, 17 patients were female and 46 were males [4], out of 15 patients, 9 patients were male and 6 were females [6] and out of 24 patients, 16 patients were male and 8 were females [5]. Out of 75 patients, 51 patients were male and 24 were males [8].

Table-3] Showing sex Distribution in different series.

\begin{tabular}{|l|l|l|l|l|}
\hline $\begin{array}{l}\text { Sr. } \\
\text { no }\end{array}$ & Series & Total no of patients & Males & Females \\
\hline 1 & Kotisso [4] & 63 & $46(73.01 \%)$ & $17(26.98 \%)$ \\
\hline 2 & Wing [6] & 15 & $9(60 \%)$ & $6(40 \%)$ \\
\hline 3 & Gibson JB [5] & 24 & $16(66.6 \%)$ & $8(33.3 \%)$ \\
\hline 4 & Ellis H [8] & 75 & $51(68 \%)$ & $24(32 \%)$ \\
\hline 5 & Present study & 38 & $28(73.68 \%)$ & $10(26.31 \%)$ \\
\hline
\end{tabular}

All the studies indicate that incidence of gastric outlet obstruction secondary to chronic peptic disease is more in males [table-3].

In the present study, the mean operative time required was 108.21 minutes $(85-145 \mathrm{~min})$. The mean operating time in the study by Bin Dayna K [9] was 180 mins, by Palanivelu C [10] was 98 mins (80-124mins), by Wing [6] was 114 mins(70-180mins),by Wyman[3] was 210 mins(180- 240mins).The findings of present study are comparable to that of study $[6,10]$.

In the present study, the conversion rate was $5.26 \%$. The reason for conversion was intraoperative adhesions in a patient previously operated for peptic ulcer perforation. No conversion had been noted in study by $[6,10,11]$. They had not mentioned that whether their patients previously operated for peptic ulcer or its complication or not. Thus, we can infer that the most common reason for conversion is adhesions. In such cases surgeon should not hesitate to convert the procedure to open. 
In the study by Wyman [6], conversion to laparotomy was necessary in one patient. In their first patient conversion to laparotomy near the end of procedure was necessary after completion of the anastomosis when no lumen could be visualized on flexible endoscopy. At the laparotomy the gastrojejunostomy was opened but the original stoma was found to have been adequate, contrary to endoscopic finding. It was refashioned by hand. Conversion under these circumstances reflects sound surgical judgment and should not be considered a complication of laparoscopic gastrojejunstomy and total truncal vagotomy.

According VAS scale, the present study showed that: On day 1, the mean post operative pain was 5.26. On day 2 , the mean post operative pain was 3.On day 7 , the mean post operative pain was 0.36 . The visual analogue scale values on day 1, 2 and 7 were less. The present study findings are comparable to study by [12]. Thus, patients operated laparoscopically experience less pain, right from the day of operation because of smaller keyhole incisions.

In the present study, the mean analgesic requirement was 46.77 hours (1.94 days). The mean analgesic requirement was 49 hrs (2.01days) in [13]. Wing [6] conducted a study over 15 patients and found that the mean post operative analgesic requirement was an average $1(0-6)$ doses of intramuscular pethidine. The present study findings are comparable with the study of [13]. Thus, patients undergoing laparoscopic gastrojejunostomy require less analgesic because of smaller keyhole incisions thereby causing less pain in contrast to longer muscle cutting incision. The present study shows that the mean time for starting oral feeds was 4.73 days. Palanivelu C [7] conducted a study over 142 patients and found that the mean time to start tolerating regular diet was 5.5 days after laparoscopic gastrojejunostomy and truncal vagotomy. Wyman [3] conducted a study over 12 patients and mentioned that nasogastric tubes were removed on second or third postoperative day when drainage was minimal and soft diet was then commenced. Peristaltic sound recovery timing and anorectum exhaust timing in laparoscopic method was much shorter [12] .The present study is comparable to the study done by [7]. In the present study, the mean time for ambulation was 8.42 hours. Dulucq [14] conducted a study over 33 patients and found that the mean time required for ambulation was $2.3+/-0.7$ days. Wyman [3] found that patient mobilizes quickly on day one postoperative [table-4].

Table-4] Post Operative Hospital Stay.

\begin{tabular}{|l|l|l|l|}
\hline Sr. No. & Series & No. of patients & Postoperative hospital stay \\
\hline 1 & Bin Dayna K [9] & 01 & 42 days \\
\hline 2 & Palanivelu C[7] & 142 & 5.2 days \\
\hline 3 & Harrel A G [15] & 14 & 5 days \\
\hline 4 & Mohammed Al [13] & 21 & 3 days \\
\hline 5 & Wing [6] & 15 & 12.9 days \\
\hline 6 & Wyman [3] & 12 & 6 days \\
\hline 7 & Dulucq [14] & 33 & 3 days \\
\hline 8 & Present study & 38 & 6.52 days \\
\hline
\end{tabular}

The present study is comparable with the studies of other authors $[3,4,7]$. The keyhole size incisions, less post operative pain score, early ambulation with early start of oral feeds and shorter convalescence allow early discharge from hospital.

The present study shows the incidence of intraoperative complication of suture line bleeding was $5.26 \%$. Suture line bleeding as a complication is seen in $1.66 \%$ of the patients in analysis by [7]. The hemorrhage from the staple line was controlled by placing a continuous running suture of 3-0 polygalactine over staple line. Incidence of no intraoperative complication of anastomotic gapping was observed in the present study. Anastomotic gapping as a complication was seen in one case $(0.83 \%)$ in analysis of [7]. Endo GIA stapler was applied on wrongly on the jejunal mesentry side, they could identify gap immediately and sutured it with polygalactine 3-0. Abdel Salam [11] found the incidence to be $5.55 \%$. In the Present study, there was no mortality seen. The incidence of Intraoperative mortality in study $[3,6,7,11]$ was also found to be zero.

yman [3] in their study found the incidence $16.66 \%$ i.e. 2 out of 12 patients. One patient remains hospitalized for 41 days due to delayed gastric emptying. This resolved on conservative management with parenteral nutrition and nasogastric drainage .One patient was readmitted 2 weeks following surgery with recurrent vomiting due to gastric stasis. This resolved on conservative management without recourse to parenteral nutrition. Both patients with gastric stasis underwent contrast studies that showed no mechanical obstruction. The present study shows no incidence of postoperative efferent loop kinking. Delayed gastric emptying as a complication seen in 4 patients $(26.66 \%)$ and were treated conservatively and able to be discharged from hospital on postoperative day 13, 25, 31 and 32 respectively [6]. The present study shows the incidence of delayed gastric emptying to be $10.52 \%$.It was manifested by Ryle tube discharge of more than $500 \mathrm{ml}$ daily with upper abdominal distension and vomiting on oral intake despite good peristalsis. They were managed conservatively with prolonged gastric decompression and intravenous support. Intravenous administration of metocloparamide every third day and parenteral alimentation was carried out. Their gastric paralysis resolved on tenth and twelfth postoperative days, respectively. Subsequently they had no problems with follow-up. 
Postoperative efferent loop kinking as a complication was seen in $1.66 \%$ of patients ( 2 out of 120 ) in the analysis by [7] , presenting with bilious vomiting on the $10^{\text {th }}$ and $14^{\text {th }}$ postoperative days ,respectively. Both underwent laparotomy and adhesiolysis with release of kinked loop. Both recovered well without further morbidity.The patients were discharged as soon as they could tolerate oral diet and were comfortable. In one patient has intestinal obstruction postoperatively. His length of hospitalization was 42 days. As compared to other studies sample size was very small i.e. only 1 , so incidence of postoperative intestinal obstruction very high i.e. $100 \%$. [9]. Wing [6] in their study found that, one of the patient (6.66\%) complicated with delayed gastric emptying presented one year after operation with anastomotic stenosis, which was managed by revision of the gastrojejunostomy. The present study shows no incidence of anastomotic stenosis.

The present study shows the incidence of stomal ulcer to be $5.26 \%$. Stomal ulcer as a complication was seen in $13.33 \%$ patients in the analysis by [6]. One of these patients also has history of postoperative delayed gastric emptying and he presented with perforated stomal ulcer 5 weeks after the initial operation, and a gastrectomy was performed. Wyman [3] found the incidence to be $8.33 \%$ patient and developed peritonitis 5 weeks following surgery. At laparotomy perforated stomal ulcer was found. A polya gastrectomy was performed and he made uneventful recovery.

The present study shows the incidence of postvagotomy diarrhea $10.52 \%$. The patients were treated with loperamide three times daily and all of them responded. Postoperative diarrhea was seen in $10 \%$ of patients who had undergone bilateral truncal vagotomy (obstruction group) in the analysis by [7]. The frequency of diarrhea varied from 4 to 9 times a day. The patients were treated with loperamide b.i.d. and t.i.d. as required and all of them responded. Loperamide could be discontinued after an average of 19 days (range; 8 to $45 \mathrm{~d}$ ). The incidence of postvagotomy diarrhea was zero [3]. In the the present study, there was no dumping and portsite hernia seen as a postoperative complication. The incidence of dumping and portsite hernia in study by $[3,7]$ was also found to be zero\%.

Average follow up duration in studies by Abdel salam [11] , Palanivelu C[7], and Wing [6] was $22.8 \pm$ 9.8 months, 6.4 years, 80 months ( 2 to 123 months) and 6 months (1 to 12 months) respectively. In the present study average follow up duration was $17.05 \pm 7.04$ months.

\section{Conclusion: -}

It is viable and safe option with shorter operative time and length of stay. It can be performed successfully with minimal morbidity and no mortality. However extreme care and skill is required to identify anatomy and handling of stapler. Further experience and long term results of this approach are required before it can be advocated as routine surgical practice.

\section{References:-}

[1]. Chung SCS (2003) Current management of acute gastrointestinal bleeding. Scand J Gastroenterol Suppl. 237 ; 9-12.

[2]. Cushieri A, Giles G.R (1995) The stomach and Duodenum , Moossa's Essential Surgical Practice, Butter Worth - Heneman Ltd., Linacre House, Jordan Hill, Oxford. Third Edition,721-728.

[3]. Wyman A, Miles-D.R (1996 laparoscopic truncal vagotomy and gastroenterostomy for pyloric stenosis; The American journal of surgery. 171(6); 600-603.

[4]. Kotiss B (2008) Gastric outlet obstruction in northwestern Ethiopia; East and Central African Journal of Surgery.5 (2); 23 -25.

[5]. Gibson JB, Behrman SW(2000 ) Gastric outlet obstruction resulting from peptic ulcer disease requiring surgical intervention is infrequently associated with Helicobacter pylori infection; J Am coll Surg .191(1):32-7.

[6]. Wing T. S (2004) Vagotomy and gastrojejunostomy for benign Gastric Outlet Obstruction; journal of laparoendoscopic and advanced surgical techniques.14 (5); 12-15.

[7]. Palanivelu C (2007) Art of Laparoscopic Surgery Textbook and Atlas: jayvee brother's medical publishers (P) ltd. volume 1:413417.

[8]. Perng C, Lin H, Lo W, et al (1996) Characteristics of patients with benign gastric outlet obstruction requiring surgery after endoscopic balloon dilation. Am J Gastroenteral. 91; 987-980.

[9]. Bin Dayna k, Board G, Al Bareeq R (2007) Laparoscopic Vagotomy: Feasible Option for the Chronic Peptic Ulcer Patient; Bahrain Medical Bulletin, 29(4); 190-193.

[10]. Palanivelu C (2006) Lapaoscopic management of acid peptic disease; surgical laparoscopy, endoscopy \& percutaneous techniques. 16(5): 312-316.

[11]. Abdel-Salam, Katri KM et al (2009) Laparoscopic Assisted Truncal Vagotomy and Gastrojejunostomy: Trial of Simplification: Journal of Laparoendoscopic and Advanced Surgical Techniques.19 (2): 125-127.

[12]. Geng W, Cao Y, Chang Y et al (1999) Recovery of gastrointestinal motility following laparoscopic versus open cholecystectomy. 37(7):415-417.

[13]. Mohammed Al-Rashedy, Dadibhai M, Shareif A (2005) Laparoscopic gastric bypass for gastric outlet obstruction is associated with smoother, faster recovery and shorter hospital stay compared with open surgery. J.Hepatobilliary pancreas, surg 12: 474-478.

[14]. Dulucg JL (2005) Completely laparoscopic total and partial gastrectomy for benign and malignant diseases: A single institute's prospective analysis; Journal of the American college of surgeons. 200(2); 191-197.

[15]. Harrel AG, Sing RF, NovitskyYW(2006) Re-evaluation of Vagotomy and Gastrojejunostomy for Benign Gastric Outlet obstruction; Journal of Laparoendoscopic and Advanced Surgical Techniques. 16(1):161-169.

[16]. Lee FY, Leung KL, Lai BS et al (2001).predicting mortality and morbidity of patients operated on for perforated peptic ulcers. Arch Surg.136; 90-94. 